\title{
Erratum to: Is ursodeoxycholic acid crucial for ischemia/ reperfusion-induced ovarian injury in rat ovary?
}

\author{
Ali Akdemir • Cagdas Sahin • Oytun Erbas • \\ Ahmet O. Yeniel • Fatih Sendag
}

Published online: 25 February 2015

(c) Springer-Verlag Berlin Heidelberg 2015

\section{Erratum to: Arch Gynecol Obstet \\ DOI 10.1007/s00404-015-3646-9}

Unfortunately, the third author Oytun Erbas's university has been published with typos in the official online publication. The correct university name is given in the affiliation.

The online version of the original article can be found under doi:10.1007/s00404-015-3646-9.

\footnotetext{
A. Akdemir · A. O. Yeniel · F. Sendag

Department of Obstetrics and Gynecology, Medicine Faculty,

Ege University, 35040 Izmir, Turkey

C. Sahin $(\bowtie)$

Department of Obstetrics and Gynecology, Tepecik

Training and Research Hospital, Fevzi Cakmak Caddesi.

No: 13/6 Bornova, 35170 Izmir, Turkey

e-mail: cagdasdr@yahoo.com

O. Erbas

Department of Physiology, Medicine Faculty,

Bilim University, 34394 Istanbul, Turkey
} 